\title{
Correlations of MMP-1, MMP-3, and MMP-12 with the degree of atherosclerosis, plaque stability and cardiovascular and cerebrovascular events
}

\author{
WEI HU ${ }^{1}$, RUI WEI ${ }^{2}$, LIYUE WANG ${ }^{3}$, JINGQIAN LU ${ }^{4}$, \\ HONGMING LIU ${ }^{1}$ and WEI ZHANG ${ }^{2}$
}

Departments of ${ }^{1}$ Cardiology and ${ }^{2}$ Emergency, The First Affiliated Hospital of Kunming Medical University,
Kunming, Yunnan 650000; ${ }^{3}$ Department of Cardiology, Wuhan Puren Hospital, Wuhan, Hubei 430000;
${ }^{4}$ Department of Cardiology, Kunming Calmette International Hospital, Kunming, Yunnan 650000, P.R. China

Received June 20, 2017; Accepted October 6, 2017

DOI: $10.3892 /$ etm.2017.5623

\begin{abstract}
We analyzed the effects of matrix metalloproteinase (MMP)-1, MMP-3, and MMP-12 on the degree of carotid atherosclerosis (CAS) and plaque stability, and investigated their correlations with cardiovascular and cerebrovascular events (CCEs). Two hundred CAS patients were enrolled. Carotid intima-media thickness (IMT) was measured using ultrasonic examination. Patients were divided into the no plaque group (NP group), stable plaque group (SP group), and vulnerable plaque group (VP group). The Crouse method was used for the evaluation of plaque scores. Additionally, 60 healthy subjects were enrolled as the control group. Serum triacylglycerol (TG), total cholesterol (TC), low density lipoprotein cholesterol (LDL-C), and high density lipoprotein cholesterol (HDL-C) were analyzed. The serum protein levels of MMP-1, MMP-3, and MMP-12 were measured by western blotting. The frequency of CCEs within 2 years was recorded, and its correlation with MMP-1, MMP-3, and MMP-12 was analyzed. The CAS plaque scores in the SP and VP groups were significantly increased compared with the NP group, and the difference between the SP and VP groups was significant. The levels of TC, TG, LDL-C, and HDL-C of CAS patients were significantly increased compared with those in the control group, but the differences in these indexes between the patient groups were not significant. Western blotting showed that the levels of MMP-1, MMP-3, and MMP-12 in the patient groups were significantly increased compared with those in the control group, and the protein levels in the VP group were significantly higher than those in the SP and NP groups.
\end{abstract}

Correspondence to: Dr Wei Zhang, Department of Emergency, The First Affiliated Hospital of Kunming Medical University, 295 Xichang Road, Kunming, Yunnan 650000, P.R. China

E-mail: mengzhgroup@aliyun.com

Key words: MMP-1, MMP-3, MMP-12, atherosclerosis, plaque stability, cardiovascular and cerebrovascular events
Additionally, the levels of MMP-1, MMP-3, and MMP-12 had significantly positive correlations with the occurrence of CCEs in CAS patients. In conclusion, MMP-1, MMP-3, and MMP-12 are positively correlated with CCEs in CAS patients. They can be used as markers for the clinical diagnosis and prognosis of CAS.

\section{Introduction}

Atherosclerosis (AS) is generally caused by abnormal lipid metabolism, which often occurs in large and medium arterial intimal-medial vascular walls, such as in the carotid artery and coronary artery. The occurrence and development of AS result from the combined activity of various environmental and genetic factors. AS is a long-term and slowly progressive degenerative inflammatory disease (1). In general, the pathogenesis of AS is highly complex, and ultimately leads to arterial wall thickening and hardening, and lumen stenosis, ultimately blocking blood vessels and causing various cardiovascular and cerebrovascular events (CCEs). AS is the pathogenic basis of various CCEs (such as stroke and coronary heart disease) (2). In recent years, studies have shown that plaque stability in AS plays an important role in its pathogenesis (3), while inflammatory, coagulation, immune and other factors play important roles in the transformation process from stable to vulnerable plaques (4). Generally, the formation of vulnerable plaques promotes the occurrence of various CCEs.

Atherosclerotic plaques are generally composed of a lipid core covered with a fibrous cap, the latter of which primarily consists of extracellular matrix (ECM) containing a large number of collagen fibers, smooth muscle cells (SMCs), and a small number of macrophages. The lipid core consists of macrophages, SMCs, and ECM with a large number of new blood vessels in the outer membrane and plaque. Atherosclerotic plaques are classified as stable plaques and vulnerable plaques, the latter of which manifest as fibrous cap ulcers, plaque rupture, and intra-plaque hemorrhage, providing a basis for the occurrence of AS. Plaque instability is characterized by increased extracellular lipid content in the plaque, and excessive number of macrophages in the fibrous 
cap. Plaque rupture increases the number of macrophages in the fibrous cap, which release a large number of proteolytic enzymes and inflammatory factors.

Matrix metalloproteinases (MMPs) are primarily released by macrophages. The MMP family of proteins is large and has different functions. The degradation products of MMPs include collagen, gelatin, ECM, and proteoglycans. Recently, a new membrane-type MMP was identified (5). Activated MMPs have higher affinity for ECM, and can degrade various ECM components and weaken the connective tissue of fibrous caps, thereby enhancing plaque instability. Moreover, MMPs are involved in tissue remodeling, inflammation, tumor invasion, wound healing, and other physiological processes (6). Studies have shown that MMP-2 and MMP-9 can promote plaque rupture and induce apoptosis of vascular SMCs through degradation of ECM and abrogation of cell-matrix interactions (7). MMP-1 promotes plaque expansion, rupture, and hemorrhage through degradation of intra-plaque collagen, providing the conditions for AS (8). In rabbits with the advanced stage of AS, the upregulation of MMP-1, MMP-3, and MMP-9, and the downregulation of MMP-14 contribute to angiogenesis in plaques and increased plaque instability (9). The aim of the present study was to investigate the effects of MMP-1, MMP-3, and MMP-12 on the degree of carotid atherosclerosis (CAS) and plaque stability, and their correlations with CCEs, to screen for new markers for the clinical diagnosis of CAS.

\section{Patients and methods}

Patients. Two hundred patients diagnosed with CAS in the First Affiliated Hospital of Kunming Medical University from January 2015 to October 2016 were selected, including 158 males and 42 females aged 50-72 years. In addition, 60 healthy subjects were selected, including 40 males and 20 females aged 53-72 years. CAS was evaluated in all subjects using carotid artery color Doppler ultrasonography. According to the results of this evaluation, the patients were divided into the no plaque group (NP group, $n=63$, including 47 males and 14 females aged $60.32 \pm 6.78$ years), stable plaque group (SP group, $n=69$, including 54 males and 11 females aged $66.45 \pm 8.68$ years), and vulnerable plaque group (VP group, $\mathrm{n}=68$, including 57 males and 17 females aged $59.92 \pm 9.46$ years), and the healthy subjects were set as the control group. There were no significant differences in sex ratio or age between the groups $(\mathrm{P}>0.05)$. This study was approved by the Institutional Research Ethics Committee of Kunming Medical University. All study participants have signed informed consent forms.

Reagents. Serum total cholesterol (TC) assay kit, serum triacylglycerol (TG) assay kit, serum low density lipoprotein cholesterol (LDL-C) assay kit, serum high density lipoprotein cholesterol (HDL-C) assay kit (Beijing Solarbio Life Sciences Co., Ltd., Beijing, China); rabbit anti-human MMP-1, MMP-3, MMP-12, and $\beta$-actin primary polyclonal antibody (dilution, 1:500; cat. nos. 13130, 14351 and 94808), horseradish peroxidase (HRP)-conjugated goat anti-rabbit secondary polyclonal antibody (dilution, 1:1,000; cat. no. 7074; Cell Signaling Technology, Beverly, MA, USA); modified BCA kit (Shanghai Sangon biotech Co., Ltd., Shanghai, China).

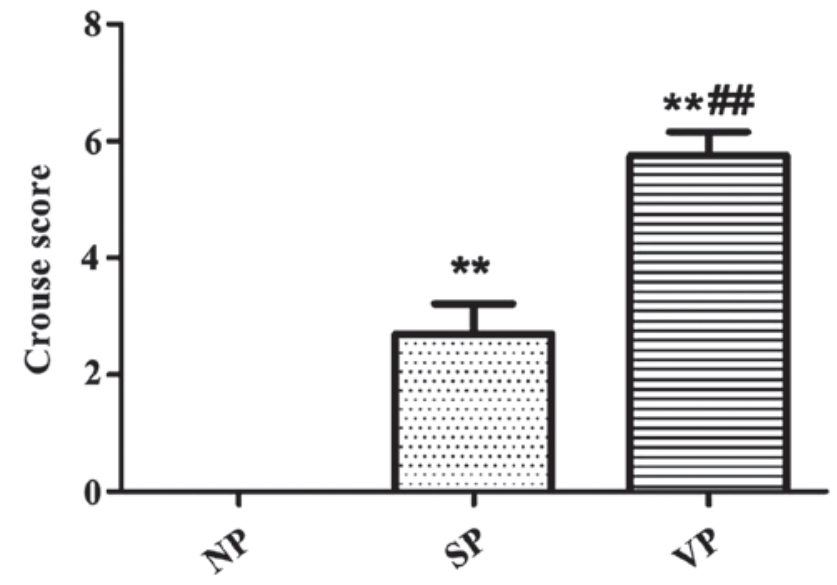

Figure 1. Crouse scores of CAS patients in each group. The plaque scores in the SP and VP groups were significantly increased compared with the NP (no plaque) group, and the plaque score in the VP group was significantly higher than in the SP group; ${ }^{* *} \mathrm{P}<0.01$ vs. NP; ${ }^{\# \#} \mathrm{P}<0.01$ vs. SP.

Carotid artery ultrasonography and plaque assessment. The patients underwent carotid artery ultrasonography using a Philip iE33 ultrasound diagnostic instrument. Under the supine position, the ultrasound probe scanned the head vertically and horizontally along the direction of the common carotid artery. Intima-media thickness (IMT) of carotid arteries was measured and recorded at a depth of $3 \mathrm{~cm}$. Each patient underwent detection three times with the mean IMT as the standard for evaluating the degree of CAS. IMT $>1.2 \mathrm{~mm}$ was defined as plaque formation. Carotid atherosclerotic plaques were classified according to the criteria set by the American Heart Association (10), and plaques were assessed using the Crouse method (11).

Analysis of blood lipid levels. Lipid levels in venous blood of subjects in each group were measured according to the instructions of the serum TG assay kit, serum TG assay kit, serum LDL-C assay kit, and serum HDL-C assay kit. Blood samples were centrifuged at $2500 \mathrm{x} \mathrm{g}$ at $4^{\circ} \mathrm{C}$ for $10 \mathrm{~min}$, and serum was collected and stored at $-80^{\circ} \mathrm{C}$ until use.

Analysis of serum levels of MMP-1, MMP-3, and MMP-12. A total of $4 \mathrm{ml}$ of fasting venous blood was drawn from each subject in the early morning and immediately sent to the laboratory for centrifugation at 2,609 x g for $10 \mathrm{~min}$. Serum was then collected and stored at $-80^{\circ} \mathrm{C}$. Western blotting was used to measure the serum levels of MMP-1, MMP-3, and MMP-12. A total of $40 \mu \mathrm{g}$ of total serum protein was separated by $10-15 \%$ sodium dodecyl sulfate polyacrylamide gel electrophoresis, and transferred to nitrocellulose membranes. Membranes were blocked using 5\% skim milk at room temperature for $2 \mathrm{~h}$, and incubated with rat anti-MMP-1, MMP-3, and MMP-12 and $\beta$-actin primary antibodies $(1: 1,000)$ at $4{ }^{\circ} \mathrm{C}$ overnight. The following day, membranes were washed three times with $0.05 \%$ TRIS-buffered saline-Tween, and incubated with HRP-conjugated rabbit anti-mouse secondary antibody $(1: 2,000)$ at room temperature for $2 \mathrm{~h}$, followed by color development using an ECL detection system (Thermo Fisher Scientific, Waltham, MA, USA). Gray values were calculated using ImageJ software (National Institutes of Health, 

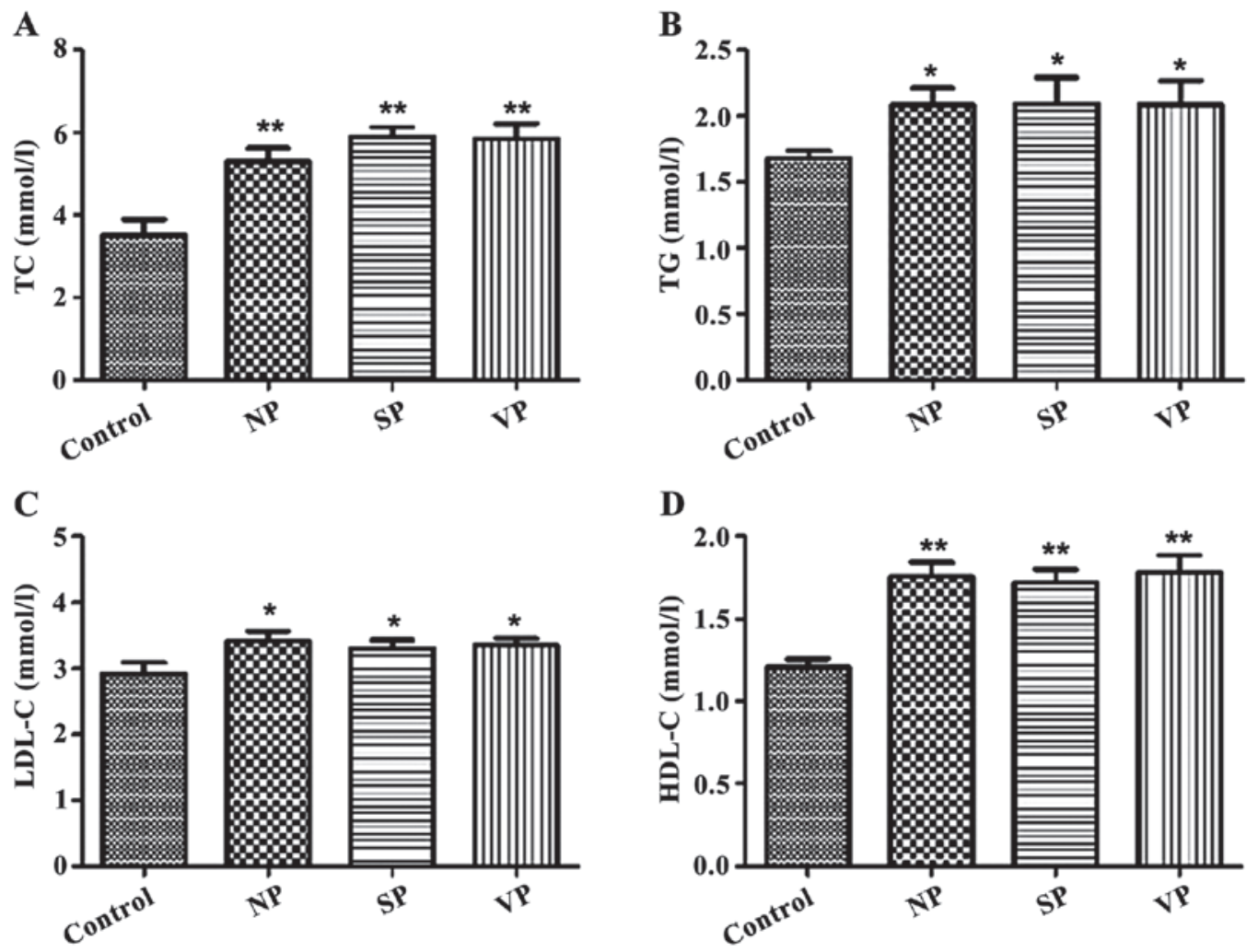

Figure 2. Blood lipid levels in each group: (A) TC, (B) TG, (C) LDL-C, (D) HDL-C. The blood lipid levels in the NP, SP, and VP groups were significantly increased compared with those in the control group, although the differences in these indexes between the NP, SP, and VP groups were not significant; ${ }^{*} \mathrm{P}<0.05$ vs. Cont; ${ }^{* *} \mathrm{P}<0.01$ vs. Cont.

Bethesda, MD, USA). Target protein levels were normalized to the levels of $\beta$-actin.

Analysis of the correlation between MMPs and CCEs. CCEs refer to acute idiopathic diseases caused by cardiac and cerebrovascular lesions under the action of risk factors, and include sudden death, acute myocardial infarction, acute cerebral infarction, cerebral hemorrhage, cerebral embolism, and cerebral thrombosis. The frequency of CCEs in CAS patients within 2 years was recorded, and its correlation with MMP-1, MMP-3, and MMP-12 was analyzed.

Statistical analysis. Statistical analysis was performed using SPSS 17.0 software (SPSS, Chicago, IL, USA). Data are presented as mean \pm standard deviation. One-way analysis of variance was performed between the groups. Linear regression analysis was performed for the correlation among indexes, and the correlation coefficient was tested using the t-test. $\mathrm{P}<0.05$ was considered to indicate a statistically significant difference.

\section{Results}

Plaque grouping and plaque scores of CAS patients. The plaques of CAS patients were divided into the no plaque group (NP group, $n=63$ ), stable plaque group (SP group, $n=69$ ), and vulnerable plaque group (VP group, $n=68$ ) according to the criteria. The Crouse method was used for the evaluation of plaque score in each group. The plaque scores in the SP and VP groups were significantly increased compared with the
NP group, and the plaque score in VP group was significantly higher than in the SP group (Fig. 1).

Analysis of serum lipid levels. The serum levels of TC, TG, LDL-C, and HDL-C in each group were measured. The levels of TC, TG, LDL-C, and HDL-C in the patient groups (NP, SP, and VP) were significantly increased compared with those in healthy subjects $(\mathrm{P}<0.05)$ (control). However, the differences in these indexes between the patient groups were not significant $(\mathrm{P}>0.05)$ (Fig. 2).

Comparisons of protein levels of serum MMP-1, MMP-3, and MMP-12. The serum levels of MMP-1, MMP-3, and MMP-12 protein in each group were measured by western blotting (Fig. 3). The protein levels of serum MMP-1, MMP-3, and MMP-12 in the patient groups (NP, SP, and VP) were significantly increased compared with those in the control group. The protein levels of MMP-1, MMP-3, and MMP-12 in the VP group were increased compared with those in the NP and SP groups, and the differences were significant, demonstrating that MMP-1, MMP-3, and MMP-12 play important roles in carotid atherosclerotic plaque stability.

Analysis of the correlation between MMPs and CCEs. The frequency of CCEs in CAS patients within 2 years was recorded, and its correlation with MMP-1, MMP-3, and MMP-12 levels was analyzed (Fig. 4). MMP-1 level was positively correlated with CCEs $\left(\mathrm{R}^{2}=0.7535\right)$ (Fig 4A); MMP-3 level was positively correlated with CCEs ( $\left.\mathrm{R}^{2}=0.6721\right)$ (Fig. 4B); and MMP-12 level was positively correlated with $\mathrm{CCEs}\left(\mathrm{R}^{2}=0.5567\right)$ (Fig. $\left.4 \mathrm{C}\right)$; 
A

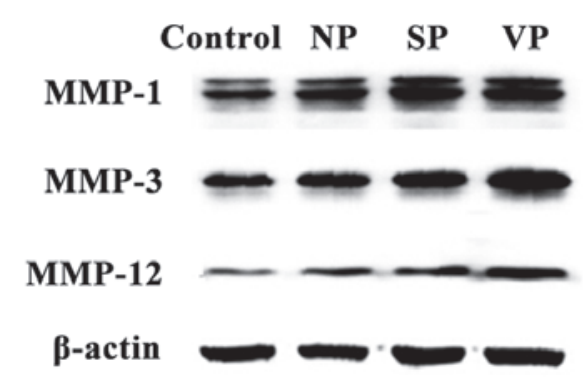

B

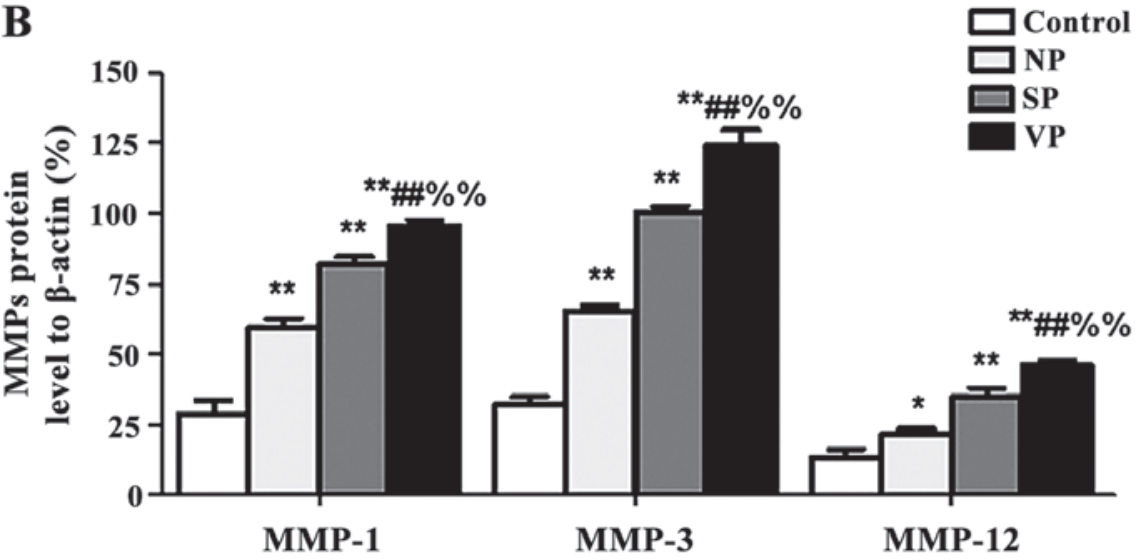

Figure 3. Analysis of serum levels of MMP-1, MMP-3, and MMP-12 protein in each group. (A) Western blot showed that the serum levels of MMP-1, MMP-3, and MMP-12 in the NP, SP, and VP groups were significantly increased compared with those in the control group; (B) the protein levels in the VP group were significantly higher than those in the SP and NP groups; ${ }^{*} \mathrm{P}<0.05$ vs. Cont; ${ }^{* *} \mathrm{P}<0.01$ vs. Cont; ${ }^{\# \#} \mathrm{P}<0.01$ vs. NP; ${ }^{\%} \mathrm{P}<0.01$ vs. SP.

$\mathbf{A}$

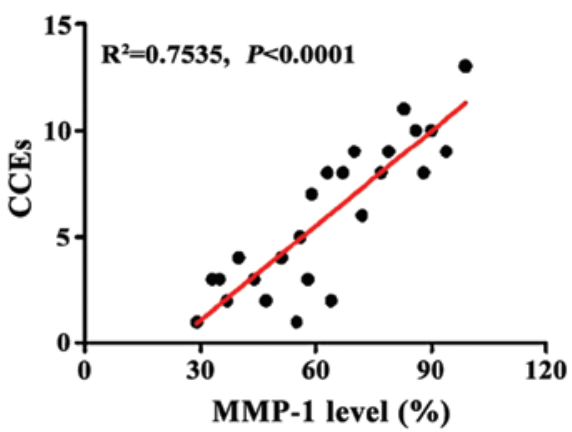

B

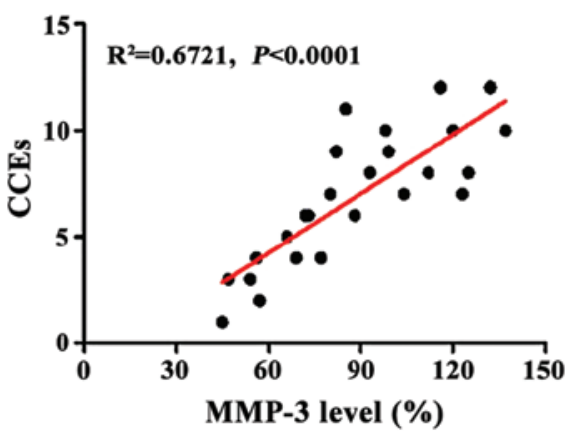

C

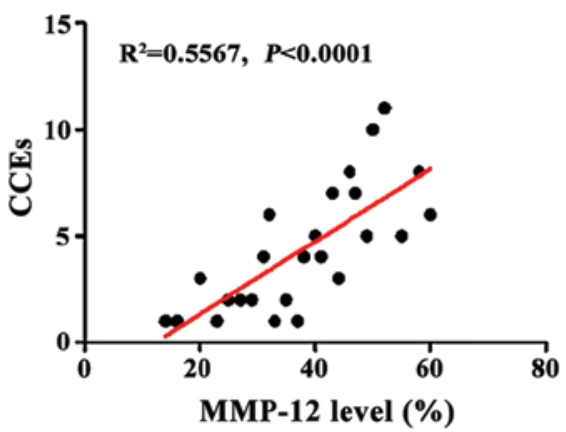

Figure 4. The correlation between MMPs and CCEs. (A) Analysis of the correlation between MMP-1 and CCEs; (B) analysis of the correlation between MMP-3 and CCEs; (C) analysis of the correlation between MMP-12 and CCEs. The results of linear regression analysis showed that there is a significantly positive correlation between MMPs and CCEs.

and the differences in the correlations of the three groups were highly significant $(\mathrm{P}<0.0001)$.

\section{Discussion}

Studies have shown that CAS patients suffer from common carotid artery intimal thickening. Plaques tend to be vulnerable, and the incidence rate is significantly higher than that in the healthy group (12). The transformation process from stable atherosclerotic plaques into vulnerable plaques is highly complex, involving inflammatory, immune, and clotting factors, among which the inflammatory response plays an important role in plaque vulnerability. In the pathogenesis of CAS, stable plaques are affected by hemodynamic factors, various inflammatory factors, and increased arterial wall stress in the lumen, causing plaque rupture and hemorrhage, and ulcer formation. The lipid core and collagen fibers can activate platelets and initiate the blood clotting response, leading to thrombosis within the arteries, or plaque shedding, forming microemboli and blocking blood vessels which results in the decreased blood flow associated with CCEs (13). In recent years, studies have shown that angiogenesis plays an important role in the development and progression of AS, and plaque rupture is closely related to increased microvessel formation (14). Studies have also reported that the number of nutrient vessels within vulnerable plaques is 2-4 times higher than in stable plaques, and CAS plaques and their stability are strongly correlated with angiogenesis (15).

MMPs are an ion-dependent (such as $\mathrm{Zn}^{2+}$ and $\mathrm{Ca}^{2+}$ ) neutral protease family. They are involved in the pathogenesis of AS. MMPs are primarily released by macrophages, but are expressed in SMCs and endothelial cells to a certain extent. MMP-1 can degrade type I and type III collagen, which is conducive to plaque expansion, rupture, and thrombosis (16). MMP-3 can degrade proteoglycans and partial collagen in principle, and plays an important role in the activation of MMPs. MMP-12 is a stromelysin that can promote the progression of AS by degrading ECM components. Studies have shown that loss of MMP-1 in lung cancer cells can significantly reduce the incidence of AS through the protease-activated receptor-1 pathway (17), and MMP-1 and MMP-3 can degrade innermembrane glycans and release fibroblast growth factors (18). Serum levels of MMP-3 in patients with myocardial infarction are decreased, and the level of MMP-3 is negatively correlated with atherosclerotic plaque region (19). MMP-12 can degrade ECM to accelerate the incidence of AS, and the overexpression of MMP-12 in transgenic rabbits can increase the expression of MMP-1 and MMP-3, indicating that MMP-12 plays a 
significant role in the series activation of MMPs (20). MMPs therefore play important roles in the pathogenesis and degree of plaque stability in AS.

We found that the Crouse scores of vulnerable plaques were significantly higher than those associated with no plaques and stable plaques, and the blood lipid levels of CAS patients were significantly higher than those in healthy subjects. The levels of MMP-1, MMP-3, and MMP-12 in subjects with vulnerable plaques were significantly increased compared with those in subjects with no plaques and stable plaques. Therefore, it is likely that MMP-1, MMP-3, and MMP-12 can increase plaque instability and promote the transformation from stable to vulnerable plaques to accelerate the occurrence and development of CAS. In addition, MMP-1, MMP-3, and MMP-12 showed significantly positive correlations with the pathogenesis of CCEs, indicating that they can be used as new indexes for evaluating the severity of CAS and the occurrence of CCEs as well as clinical markers for the prognosis, diagnosis, and treatment of CAS. In conclusion, this study provided a new theoretical basis for screening for new targets in the diagnosis of CAS.

\section{References}

1. Ross R: The pathogenesis of atherosclerosis: A perspective for the 1990s. Nature 362: 801-809, 1993.

2. Singh RB, Mengi SA, Xu Y-J, Arneja AS and Dhalla NS: Pathogenesis of atherosclerosis: A multifactorial process. Exp Clin Cardiol 7: 40-53, 2002.

3. Naghavi M, Libby P, Falk E, Casscells SW, Litovsky S, Rumberger J, Badimon JJ, Stefanadis C, Moreno P, Pasterkamp G, et al: From vulnerable plaque to vulnerable patient: a call for new definitions and risk assessment strategies: Part I. Circulation 108: 1664-1672, 2003.

4. Hansson GK and Hermansson A: The immune system in atherosclerosis. Nat Immunol 12: 204-212, 2011.

5. Nagase $\mathrm{H}$ and Woessner JF Jr: Matrix metalloproteinases. J Biol Chem 274: 21491-21494, 1999.

6. Verma RP and Hansch C: Matrix metalloproteinases (MMPs): Chemical-biological functions and (Q)SARs. Bioorg Med Chem 15: 2223-2268, 2007.

7. Pasterkamp G, Schoneveld AH, Hijnen DJ, de Kleijn DP, Teepen H, van der Wal AC and Borst C: Atherosclerotic arterial remodeling and the localization of macrophages and matrix metalloproteases 1,2 and 9 in the human coronary artery. Atherosclerosis 150: 245-253, 2000.

8. Welgus HG, Campbell EJ, Bar-Shavit Z, Senior RM and Teitelbaum SL: Human alveolar macrophages produce a fibroblast-like collagenase and collagenase inhibitor. J Clin Invest 76: 219-224, 1985.
9. Liu XQ, Mao Y, Wang B, Lu XT, Bai WW, Sun YY, Liu Y, Liu HM, Zhang L, Zhao YX, et al: Specific matrix metalloproteinases play different roles in intraplaque angiogenesis and plaque instability in rabbits. PLoS One 9: e107851, 2014.

10. Stary HC: Natural history and histological classification of atherosclerotic lesions: An update. Arterioscler Thromb Vasc Biol 20: 1177-1178, 2000.

11. Crouse JR, Harpold GH, Kahl FR, Toole JF and McKinney WM: Evaluation of a scoring system for extracranial carotid atherosclerosis extent with B-mode ultrasound. Stroke 17: 270-275, 1986.

12. Touboul PJ, Elbaz A, Koller C, Lucas C, Adraï V, Chédru F, Amarenco P and Investigators G; The GENIC Investigators: Common carotid artery intima-media thickness and brain infarction : The Etude du Profil Génétique de l'Infarctus Cérébral (GENIC) case-control study. Circulation 102: 313-318, 2000.

13. Cho YP, Kwon TW and Kim GE: Sonographic appearance of a free-floating atheromatous plaque in a patient with acute stroke. J Clin Ultrasound 30: 317-321, 2002.

14. Battegay EJ: Angiogenesis: Mechanistic insights, neovascular diseases, and therapeutic prospects. J Mol Med (Berl) 73: 333-346, 1995.

15. Kolodgie FD, Virmani R, Burke AP, Farb A, Weber DK, Kutys R, Finn AV and Gold HK: Pathologic assessment of the vulnerable human coronary plaque. Heart 90: 1385-1391, 2004.

16. Galis ZS, Muszynski M, Sukhova GK, Simon-Morrissey E, Unemori EN, Lark MW, Amento E and Libby P: Cytokinestimulated human vascular smooth muscle cells synthesize a complement of enzymes required for extracellular matrix digestion. Circ Res 75: 181-189, 1994.

17. Foley CJ, Fanjul-Fernández M, Bohm A, Nguyen N, Agarwal A, Austin K, Koukos G, Covic L, López-Otín C and Kuliopulos A: Matrix metalloprotease 1a deficiency suppresses tumor growth and angiogenesis. Oncogene 33: 2264-2272, 2014.

18. Whitelock JM, Murdoch AD, Iozzo RV and Underwood PA: The degradation of human endothelial cell-derived perlecan and release of bound basic fibroblast growth factor by stromelysin, collagenase, plasmin, and heparanases. J Biol Chem 271: 10079-10086, 1996.

19. Samnegård A, Silveira A, Lundman P, Boquist S, Odeberg J, Hulthe J, McPheat W, Tornvall P, Bergstrand L, Ericsson CG, et al: Serum matrix metalloproteinase- 3 concentration is influenced by MMP-3 $-16125 \mathrm{~A} / 6 \mathrm{~A}$ promoter genotype and associated with myocardial infarction. J Intern Med 258: 411-419, 2005.

20. Liang J, Liu E, Yu Y, Kitajima S, Koike T, Jin Y, Morimoto M, Hatakeyama K, Asada Y, Watanabe T, et al: Macrophage elastase (MMP-12) accelerates the progression of atherosclerosis in transgenic rabbits. FASEB 20: A12-A13, 2006. International (CC BY-NC-ND 4.0) License. 